\title{
ON THE INTEGRAL HODGE AND TATE CONJECTURES OVER A NUMBER FIELD
}

\author{
BURT TOTARO \\ DPMMS, Wilberforce Road, Cambridge CB3 0WB, England \\ UCLA Department of Mathematics, Box 951555, Los Angeles, CA 90095-1555, USA; \\ email: totaro@math.ucla.edu
}

Received 29 November 2012; accepted 14 June 2013

\begin{abstract}
Hassett and Tschinkel gave counterexamples to the integral Hodge conjecture among 3-folds over a number field. We work out their method in detail, showing that essentially all known counterexamples to the integral Hodge conjecture over the complex numbers can be made to work over a number field.
\end{abstract}

2010 Mathematics Subject Classification: 14C30 (primary); 11F80 (secondary)

\section{Introduction}

In seeking to understand the Hodge conjecture, it is important to ask how the integral Hodge conjecture can fail. There are two known ways of producing counterexamples to the integral Hodge conjecture: Atiyah-Hirzebruch's topological approach [2,30], and Kollár's use of degenerations [16]. AtiyahHirzebruch's method gives varieties defined over a number field, but has the disadvantage that it only gives varieties of dimension at least seven. Kollár's approach disproves the integral Hodge conjecture for 3-folds, but is inexplicit in that the examples are very general complex hypersurfaces of certain degrees. Since 'very general' excludes countably many lower-dimensional subsets of the space of hypersurfaces, it was not known whether the integral Hodge conjecture was true for the countably many 3 -folds which are defined over number fields. 
By refining Kollár's method, Hassett and Tschinkel recently succeeded in disproving the integral Hodge conjecture for 3-folds over number fields [6, Remarque 5.10]. The method is flexible and should apply to other problems where one hopes to show that 'very general' behavior also occurs over number fields. The basic idea is to specialize cycles from a variety over a number field to a variety over a finite field.

Hassett-Tschinkel's method has only been described briefly by ColliotThélene and Voisin. In this paper, we work out their method in detail in Sections 3 and 4. We find that there are versions over $\mathbf{Q}$ of all the applications of Kollár's method made by Kollár [16], Debarre, Hulek, and Spandaw [7], and Colliot-Thélene and Voisin [6, Proposition 5.8]. This often requires extra work beyond Hassett-Tschinkel's method, which we do in Sections 5 and 6. The point is that the method works well if one can construct varieties of a given type over a finite field with geometric Picard number 1 . But this is impossible in many cases. For example, Swinnerton-Dyer and Shioda observed that, on the assumption of the Tate conjecture, every smooth projective surface over a finite field with even second Betti number has geometric Picard number at least 2 [1, p. 544], [27, 5.2]. We avoid that difficulty by careful choice of the family of varieties to consider.

All known counterexamples to the integral Hodge conjecture on 3-folds, including those in this paper, involve nontorsion classes in integral cohomology. The original counterexamples by Atiyah and Hirzebruch were even-degree torsion cohomology classes on varieties of dimension at least seven. Soulé and Voisin exhibited even-degree torsion cohomology classes which are not algebraic on certain 5-folds [28].

Hassett-Tschinkel's method also gives the first counterexamples to the integral Tate conjecture for 1-cycles on a variety over $\overline{\mathbf{Q}}$, and for 1-cycles on a variety over the separable closure of $\mathbf{F}_{p}(t)$. These results are essentially optimal, since Schoen showed that the usual Tate conjecture would imply the integral Tate conjecture for 1-cycles on a variety over the separable closure of a finite field $[25,5]$. The only known counterexamples to the integral Tate conjecture over finite fields are in dimension at least seven, by Colliot-Thélène and Szamuely [5, Théorème 2.1].

\section{Notation}

A variety over a field is irreducible by definition. A curve is a variety of dimension one.

The integral Hodge conjecture for a smooth complex projective variety $X$ asserts that every element of $H^{2 i}(X, \mathbf{Z})$ whose image in $H^{2 i}(X, \mathbf{C})$ is of type $(i, i)$ is the class of an algebraic cycle of codimension $i$, that is, a $\mathbf{Z}$-linear 
combination of subvarieties of $X$. The integral Tate conjecture for a smooth projective variety $X$ over a finitely generated field $k$ asserts that, for any prime number $l$ invertible in $k$, every element of étale cohomology $H^{2 i}\left(X_{k^{\text {sep }}}, \mathbf{Z}_{l}(i)\right)$ fixed by the Galois group $\operatorname{Gal}\left(k^{\mathrm{sep}} / k\right)$ is the class of an algebraic cycle with $\mathbf{Z}_{l}$ coefficients. (As Schoen points out, the integral Tate conjecture in this form fails already for 0 -cycles on the conic curve $x^{2}+y^{2}+z^{2}=0$ over $\mathbf{Q}$ [25]. As a result, it is more interesting to consider the integral Tate conjecture over the separable closure of a finitely generated field.) The integral Tate conjecture for a smooth projective variety $X$ over the separable closure $F$ of a finitely generated field is the weaker statement that, for $k$ a finitely generated field of definition of $X$, every element of $H^{2 i}\left(X_{F}, \mathbf{Z}_{l}(i)\right)$ fixed by some open subgroup of $\operatorname{Gal}(F / k)$ is the class of an algebraic cycle over $F$ with $\mathbf{Z}_{l}$ coefficients. The usual Hodge and Tate conjectures, which may actually be true, are the analogous statements with $\mathbf{Q}$ or $\mathbf{Q}_{l}$ coefficients.

Griffiths and Harris conjectured that every curve in a very general complex 3 -fold $Y$ of degree $d \geq 6$ in $\mathbf{P}^{4}$ has degree a multiple of $d$ [14]. This would in particular disprove the integral Hodge conjecture for 1-cycles on very general hypersurfaces of any degree $d \geq 6$, since there is an element of $H^{4}(Y, \mathbf{Z}) \cong \mathbf{Z}$ of degree one (represented by a line on some hypersurfaces $Y$ ) and all of $H^{4}(Y, \mathbf{Z})$ is of Hodge type $(2,2)$. More generally, Nori conjectured that, for any smooth complex projective variety $X$ and a very general sufficiently ample hypersurface $Y$ in $X$, the restriction map $\mathrm{CH}^{i} X \rightarrow \mathrm{CH}^{i} Y$ on Chow groups should be an isomorphism for all $i<\operatorname{dim} Y$ [22, p. 368]. (To be precise, Nori conjectured this for Chow groups tensored with the rationals, but the integral statement seems plausible in view of Kollár's examples [16].)

\section{Hypersurfaces in $\mathbf{P}^{1} \times \mathbf{P}^{3}$}

This section gives Hassett and Tschinkel's proof that the integral Hodge conjecture fails for some hypersurfaces of bidegree $(3,4)$ in $\mathbf{P}^{1} \times \mathbf{P}^{3}$ over $\mathbf{Q}$ [6, Remarque 5.10]. Colliot-Thélène and Voisin showed that the integral Hodge conjecture fails for very general complex hypersurfaces of bidegree $(3,4)$ in $\mathbf{P}^{1} \times \mathbf{P}^{3}$ [6, Proposition 5.8]. We give this example first because the proof is easier than the later proofs for hypersurfaces in $\mathbf{P}^{4}$. The 3 -folds here also have the interest that their Kodaira dimension is one. This is probably the smallest possible. Indeed, Voisin has proved the integral Hodge conjecture for 3-folds which are uniruled or have trivial canonical bundle and first Betti number zero [32, Theorem 2]. Grabowski proved the integral Hodge conjecture for abelian 3 -folds [12, Section 3.1]. The problem remains open for more general 3-folds of Kodaira dimension zero. 
THEOREM 3.1. The integral Hodge conjecture for 1-cycles fails for a Zariskidense set of smooth hypersurfaces of bidegree $(3,4)$ in $\mathbf{P}^{1} \times \mathbf{P}^{3}$ over $\mathbf{Q}$.

For example, the proof shows that the integral Hodge conjecture fails for the smooth hypersurface

$$
u^{3} x_{0}^{4}+t u^{2} x_{1}^{4}+t^{2} u x_{2}^{4}+t^{3} x_{3}^{4}+2 u^{3} x_{3}^{4}-2 t^{3} x_{0}^{4}+2 u^{3} x_{2}^{4}-2 t^{3} x_{1}^{4}=0
$$

in $\mathbf{P}^{1} \times \mathbf{P}^{3}$ over $\mathbf{Q}$. In this and the later examples, the proof shows more than 'Zariski-dense': the integral Hodge conjecture fails for a positive-density subset of all hypersurfaces over $\mathbf{Q}$ of bidegree $(3,4)$, counted by height. The proof also shows that the integral Hodge conjecture fails for a set of hypersurfaces over $\mathbf{Q}$ which are dense in the space of all hypersurfaces over $\mathbf{R}$ with the classical topology.

Proof. Let $X$ be a smooth hypersurface of bidegree $(3,4)$ in $\mathbf{P}^{1} \times \mathbf{P}^{3}$ over $\overline{\mathbf{Q}}$. Suppose that $X$ specializes to the singular hypersurface

$$
\left\{\left([t, u],\left[x_{0}, x_{1}, x_{2}, x_{3}\right]\right): u^{3} x_{0}^{4}+t u^{2} x_{1}^{4}+t^{2} u x_{2}^{4}+t^{3} x_{3}^{4}=0\right\}
$$

in $\mathbf{P}^{1} \times \mathbf{P}^{3}$ over $\overline{\mathbf{F}_{p}}$ for some prime number $p$. (Similar equations have been used in Kollár [17, IV.6.4.3.1] and in [31, proof of Theorem 0.1].) Then we will show that every curve in $X$ has even degree over $\mathbf{P}^{1}$. This violates the integral Hodge conjecture for $X$, since $H_{2}(X, \mathbf{Z})$ maps isomorphically to $H_{2}\left(\mathbf{P}^{1} \times \mathbf{P}^{3}, \mathbf{Z}\right)$ by the Lefschetz hyperplane theorem, and the Hodge structure on $H_{2}(X, \mathbf{Z})$ is trivial.

Using the specialization map $\mathrm{CH}_{1}\left(X_{\overline{\mathbf{Q}}}\right) \rightarrow \mathrm{CH}_{1}\left(X_{\overline{\mathbf{F}_{p}}}\right)$ [11, Example 20.3.5], it suffices to show that every curve $C$ in $X_{\overline{\mathbf{F}_{p}}}$ has even degree over $\mathbf{P}^{1}$. It suffices to compute the degree of $C \rightarrow \mathbf{P}_{\overline{\mathbf{F}_{p}}}^{1}$ restricted to the generic point of $\mathbf{P}_{\overline{\mathbf{F}_{p}}}^{1}$, or restricted further to the field $\overline{\mathbf{F}_{p}}((t))$ of Laurent series around the point $[t, u]=[0,1]$. Thus, it suffices to show that the hypersurface

$$
\left\{\left[x_{0}, x_{1}, x_{2}, x_{3}\right]: x_{0}^{4}+t x_{1}^{4}+t^{2} x_{2}^{4}+t^{3} x_{3}^{4}=0\right\}
$$

in $\mathbf{P}^{3}$ over $\overline{\mathbf{F}_{p}}((t))$ has no rational point over any odd-degree extension $\overline{\mathbf{F}_{p}}((s))$ of $\overline{\mathbf{F}_{p}}((t))$.

If there is a rational point over an odd-degree extension, then we have Laurent series $t(s)$ and $x_{i}(s)$ over $\overline{\mathbf{F}_{p}}$ which satisfy the equation above and such that the valuation $r:=\operatorname{ord}_{s}(t)$ is odd. But the valuations of the four terms in the equation are congruent to $0, r, 2 r, 3 r(\bmod 4)$. Since $r$ is odd, these lowest degrees are all different. So the only way the four terms can add up to zero is if all are identically zero. That would imply that $x_{i}(s)=0$ for all $i$, but this does not correspond to a point in projective space. Thus our hypersurface has no rational point over any odd-degree extension of $\overline{\mathbf{F}_{p}}((t))$. 
This completes the proof that the integral Hodge conjecture fails for any smooth hypersurface of bidegree $(3,4)$ in $\mathbf{P}^{1} \times \mathbf{P}^{3}$ over $\overline{\mathbf{Q}}$ which specializes to the given singular hypersurface over $\overline{\mathbf{F}_{p}}$ for some prime number $p$. The set of such hypersurfaces over $\mathbf{Q}$, for a fixed prime number $p$, is Zariski dense in the space of all hypersurfaces of bidegree $(3,4)$.

\section{Hypersurfaces in projective space over number fields}

This section presents Hassett and Tschinkel's method of producing counterexamples to the integral Hodge conjecture for hypersurfaces in $\mathbf{P}^{4}$ over number fields [6, Remarque 5.10]. We formulate the method as the following generalization of Kollár's lemma [16] (which gives very general complex hypersurfaces).

LEMMA 4.1. Let $Y$ be an irreducible projective 3-fold over $\overline{\mathbf{F}_{p}}$ with a very ample line bundle $L$ such that $L^{3}=d$ and $L \cdot C \equiv 0(\bmod k)$ for every curve $C$ in $Y$. Assume that $k$ is not a multiple of $p$ and that $d<p$. Then there is a smooth hypersurface $X$ in $\mathbf{P}^{4}$ over $\overline{\mathbf{Q}}$ (and also a smooth hypersurface over $\mathbf{F}_{p}(t)^{\mathrm{sep}}$ ) of degree $d$ such that every curve $C$ in $X$ has

$$
6 \operatorname{deg}(C) \equiv 0(\bmod k) .
$$

In fact, the conclusion holds for a Zariski-dense set of hypersurfaces $X$ over $\overline{\mathbf{Q}}$.

Note that the integral Hodge conjecture (or the integral Tate conjecture) would imply that every smooth hypersurface in $\mathbf{P}^{4}$ over $\overline{\mathbf{Q}}$ contains a 1-cycle of degree one. So the lemma gives many counterexamples to the integral Hodge conjecture. We first present the easy case of a hypersurface of degree 64 over $\overline{\mathbf{Q}}$. With more work, Lemma 5.1 will give an example of lower degree, 48, which is defined over Q. (In view of the Griffiths-Harris conjecture, I would expect that for every $d$ at least 6 there is a smooth hypersurface $X$ of degree $d$ in $\mathbf{P}^{4}$ over $\mathbf{Q}$ such that every curve in $X_{\overline{\mathbf{Q}}}$ has degree a multiple of $d$.)

The proof of Lemma 4.1 gives something more precise than hypersurfaces over $\overline{\mathbf{Q}}$ : there is a Zariski-dense set of hypersurfaces $X$ satisfying the conclusion over any number field $F$ that has $\mathbf{F}_{p^{r}}$ as a residue field, for a certain positive integer $r$ that could be computed. In many cases, one can take $r=1$, and then there are hypersurfaces over $\mathbf{Q}$ that satisfy the conclusion.

COROLlaRY 4.2. There is a smooth hypersurface $X$ of degree 64 in $\mathbf{P}^{4}$ over $\overline{\mathbf{Q}}$ such that every curve on $X$ has even degree. In particular, the integral Hodge conjecture fails for $X$. 
Proof. Apply Lemma 4.1 to the 3 -fold $(Y, L)=\left(\mathbf{P}^{3}, O(4)\right)$ over $\overline{\mathbf{F}_{p}}$, for any prime number $p>64$.

Proof of Lemma 4.1. We begin with the following lemma on general projections, which is well known in characteristic zero. It is important for some later applications that we put no restriction on the singularities.

LEMMA 4.3. Let $Y \subset \mathbf{P}^{N}$ be a nondegenerate absolutely irreducible variety of dimension $n$ over an infinite field $F$. If $F$ has characteristic $p>0$, assume that $Y$ has degree less than $p$. Then a general linear projection of $Y$ to $\mathbf{P}^{n+1}$ over $F$ is a morphism that is (set-theoretically over $\bar{F}$ ) at most 2-to-1 in codimension one and at most 3-to-1 in codimension two.

Proof. Repeatedly reducing from $Y$ to a general hyperplane section, we can assume that $Y$ has dimension two. That is, we have to show that, for a nondegenerate surface $X$ in $\mathbf{P}^{N}$, with $\operatorname{deg}(X)<p$ if $F$ has characteristic $p$, a general projection of $Y$ to $\mathbf{P}^{3}$ is (set-theoretically) 2-to-1 at most on a one-dimensional subset, 3-to-1 at most on a zero-dimensional subset, and nowhere 4-to-1 or worse. For surfaces in characteristic zero, this is proved by Griffiths and Harris [13, pp. 611-613] using the general position lemma: for a nondegenerate curve in projective space, a general hyperplane section of the curve consists of points in linear general position. The general position lemma fails in general in characteristic $p$, but it holds for curves in $\mathbf{P}^{N}$ (possibly singular) of degree less than $p$, by Rathmann [24, Corollaries 1.8, 2.2]. Then the Griffiths-Harris argument applies.

To prove Lemma 4.1, let $\varphi: Y \rightarrow \mathbf{P}^{N}$ be the embedding given by $L$ over $\overline{\mathbf{F}_{p}}$. Let $\pi: Y \rightarrow \bar{Y} \subset \mathbf{P}^{4}$ be a general projection. By Lemma 4.3, every curve $C$ in $\bar{Y}$ is the image of a curve $D$ in $Y$ by a morphism that is set-theoretically 1-to-1, 2-to-1, or 3-to-1. It follows that the degree of the morphism $D \rightarrow C$ is $a p^{r}$ for some $1 \leq a \leq 3$ and some $r \geq 0$. Since $L \cdot D \equiv 0(\bmod k)$, we have $6 p^{r} \operatorname{deg}(C)=6 p^{r} L \cdot C \equiv 0(\bmod k)$. Since $p$ does not divide $k$, we have $6 \operatorname{deg}(C) \equiv 0(\bmod k)$.

There is a smooth hypersurface $X$ of degree $d$ in $\mathbf{P}^{4}$ over $\overline{\mathbf{Q}}$ (or over $\mathbf{F}_{p}(t)^{\mathrm{sep}}$ ) that specializes to the singular hypersurface $\bar{Y} \subset \mathbf{P}^{4}$ over $\overline{\mathbf{F}_{p}}$. For any such hypersurface, the specialization map $\mathrm{CH}_{1}(X) \rightarrow \mathrm{CH}_{1}(\bar{Y})$ [11, Example 20.3.5] shows that every curve $C$ in $X$ has $6 \operatorname{deg}(C) \equiv 0(\bmod k)$. The set of hypersurfaces $X$ over $\overline{\mathbf{Q}}$ that specialize to any given hypersurface $\bar{Y}$ over $\overline{\mathbf{F}_{p}}$ is Zariski dense in the space of all hypersurfaces, as we want. 


\section{Degree 48}

The complex 3-fold hypersurfaces of lowest degree for which the integral Hodge conjecture is known to fail have degree 48, by Kollár [16]. (Note the typographical error in the first example in [16]: these hypersurfaces have degree $3 k^{2}$ for $k \geq 4$, not $k^{2}$.) In this section, we find that the integral Hodge conjecture also fails for some hypersurfaces of degree 48 over $\mathbf{Q}$.

Kollár's example relies on the Noether-Lefschetz theorem that a very general quartic surface in $\mathbf{P}^{3}$ over $\mathbf{C}$ has Picard number 1. We want to apply Lemma 4.1, but there is an extra complication: if we believe the Tate conjecture, then every smooth surface of even degree in $\mathbf{P}^{3}$ over $\overline{\mathbf{F}_{p}}$ has Picard number at least 2, by Swinnerton-Dyer and Shioda [1, p. 544], [27, 5.2]. (In fact, we know unconditionally that smooth quartic surfaces over $\overline{\mathbf{F}_{p}}$ have Picard number at least 2 if $p \geq 3$, since the Tate conjecture has been proved for K3 surfaces in characteristic $p \geq 3$ by Nygaard-Ogus et al. [4, 19,20,23].) We get around the problem by finding a quartic surface with a node in $\mathbf{P}^{3}$ over $\mathbf{F}_{p}$ which has geometric Picard number 1 . The method was suggested by de Jong and Katz's construction of smooth surfaces of any odd degree at least five in $\mathbf{P}^{3}$ over $\overline{\mathbf{F}_{p}}$ with Picard number 1 [10, Theorem 6.11].

Lemma 5.1. There is a smooth hypersurface $X$ of degree 48 in $\mathbf{P}^{4}$ over $\mathbf{Q}$ such that every curve in $X_{\overline{\mathbf{Q}}}$ has even degree. In particular, the integral Hodge conjecture fails for $X$. The set of hypersurfaces $X$ over $\mathbf{Q}$ with these properties is Zariski dense in the space of all hypersurfaces of degree 48.

Proof. In order to get examples over $\mathbf{Q}$ rather than just $\overline{\mathbf{Q}}$, it seems that we need to use the idea of Lemma 4.1 rather than just quoting the lemma. Let $S \rightarrow B$ be the universal family of quartic surfaces in $\mathbf{P}^{3}$ with exactly one node, which we consider over $\mathbf{Z}[1 / 2]$; then $B$ is an integral scheme over $\mathbf{Z}[1 / 2]$. Let $S_{\mathbf{Q}(B)}$ be the generic fiber of $S \rightarrow B$, which is a nodal quartic over the function field $\mathbf{Q}(B)$. Let $X=S \times \mathbf{P}^{1}$ with the line bundle $L=\pi_{1}^{*} O(1) \otimes \pi_{2}^{*} O(4)$. Thus $X \rightarrow B$ is a family of 3-folds equipped with a very ample line bundle $L$ having degree $L^{3}$ equal to $3 \cdot 4^{2}=48$. Let $X_{\mathbf{Q}(B)}=S_{\mathbf{Q}(B)} \times \mathbf{P}^{1}$ be the generic fiber of $X \rightarrow B$, embedded in some projective space $\mathbf{P}^{N}$ using $L$. By Lemma 4.3, a general projection of $X_{\mathbf{Q}(B)} \subset \mathbf{P}^{N}$ to $\mathbf{P}^{4}$ is a morphism that is (set-theoretically) at most 2-to-1 in dimension two and at most 3-to-1 in dimension one. We can view this morphism as a rational map $X \rightarrow \mathbf{P}_{B}^{4}$ over $B$. After replacing $B$ by some nonempty open subset, we have a morphism $X \rightarrow \mathbf{P}_{B}^{4}$ over $B$ which is 2-to- 1 at most in dimension two and so on, over every point of $B$.

The arithmetic fundamental group $\pi_{1} B$ (with a base point in $B(\overline{\mathbf{Q}})$ ) has a monodromy representation on $H^{2}\left(S_{\overline{\mathbf{Q}}}, \mathbf{Z}_{2}(1)\right) \cong\left(\mathbf{Z}_{2}\right)^{21}$, where $S_{\overline{\mathbf{Q}}}$ is a quartic 
surface with a node over $\overline{\mathbf{Q}}$. This representation preserves the intersection form (which is nondegenerate $\left.\otimes \mathbf{Q}_{2}\right)$ and the vanishing subspace $\operatorname{ker}\left(H^{2}\left(S_{\overline{\mathbf{Q}}}, \mathbf{Z}_{2}(1)\right) \rightarrow\right.$ $\left.H^{4}\left(\mathbf{P}^{3}, \mathbf{Z}_{2}(2)\right)\right)$, giving a homomorphism $\pi_{1} B \rightarrow O\left(20, \mathbf{Z}_{2}\right)$ to an orthogonal group.

By Deligne's arguments using the Picard-Lefschetz theory, the image of the geometric fundamental group $\pi_{1} B \overline{\mathbf{Q}} \subset \pi_{1} B$ is Zariski dense in $O\left(20, \mathbf{Q}_{2}\right)$ [9, Theorem 4.4.2]. (Deligne's arguments using a Lefschetz pencil show that the monodromy of $\pi_{1} B_{\mathbf{C}}$ is either Zariski dense in $O(20, \mathbf{Q})$ or finite and irreducible. The finite case can be ruled out as follows, working over $\mathbf{C}$. Since the monodromy representation on the vanishing cohomology $\mathbf{Q}^{20}$ of $S_{\mathbf{C}}$ is irreducible, there is a unique invariant symmetric bilinear form on $\mathbf{Q}^{20}$ up to scalars, which must be positive or negative definite if the representation has finite image. But the Hodge-Riemann bilinear relations give that the intersection form has sign $(-1)^{p}$ on $H_{\text {prim }}^{p, q}(S)\left[13\right.$, p. 123]. Since $H_{\text {prim }}^{2,0}(S) \neq 0$, the monodromy must be Zariski dense in $O(20, \mathbf{Q})$, rather than finite.)

We return to the scheme $B$ over $\mathbf{Z}[1 / 2]$. Since the geometric fundamental group $\pi_{1} B \overline{\mathbf{Q}}$ is a subgroup of $\pi_{1} B$, the image of $\pi_{1} B$ is also Zariski dense in $O\left(20, \mathbf{Q}_{2}\right)$. By the Serre-Chebotarev density theorem, the conjugacy classes of the Frobenius elements Frob $_{x}$ associated to closed points $x$ in $B$ are equidistributed in $\pi_{1} B$ [26, Theorem 7]. Moreover, the closed points with residue field of prime order have density 1 among all closed points. (Closed points are counted with respect to the order of their residue field, and in this sense those of order $p^{r}$ with $r \geq 2$ are rare.) Therefore, the images of the Frobenius elements Frob $_{x}$ associated to closed points $x$ of $B$ with residue field of prime order are Zariski dense in $O\left(20, \mathbf{Q}_{2}\right)$.

By the Weil conjectures (Deligne's theorem), the characteristic polynomial of any Frobenius element Frob $_{x}$ on $H^{2}\left(\left(S_{x}\right)_{\overline{\mathbf{F}}}, \mathbf{Q}_{2}(1)\right)$ has rational coefficients [8]. So all eigenvalues are algebraic numbers of degree at most 20 over $\mathbf{Q}$. The set $S$ of roots of unity of degree at most 20 over $\mathbf{Q}$ is finite. So the locus in $\operatorname{SO}\left(20, \mathbf{Q}_{2}\right)$ of elements with an eigenvalue in $S$ is Zariski closed and not the whole group. Therefore, the previous paragraph's Zariski density statement gives that there is a closed point $x \in B$ such that the residue field has prime order $p$ and no eigenvalues of $\rho\left(\right.$ Frob $\left._{x}\right)$ on the vanishing cohomology in $H^{2}\left(S_{\overline{\mathbf{F}_{p}}}, \mathbf{Q}_{2}(1)\right)$ are roots of unity. It follows that the fiber over $x$ is a quartic surface $S_{\mathbf{F}_{p}}$ over $\mathbf{F}_{p}$ with one node which has geometric Picard number 1.

By the Lefschetz hyperplane theorem, $\operatorname{coker}\left(H^{2}\left(\mathbf{P}_{\overline{\mathbf{F}_{p}}}^{3}, \mathbf{Z}_{2}(1)\right) \rightarrow H^{2}\left(S_{\overline{\mathbf{F}_{p}}}\right.\right.$, $\left.\left.\mathbf{Z}_{2}(1)\right)\right)$ is torsion free [21, Theorem 7.1]. The first group is generated by $O(1)$. We know that the image of $\operatorname{Pic}\left(S_{\overline{\mathbf{F}_{p}}}\right)$ in étale cohomology is contained in the Q-line spanned by $O(1)$, and this torsion freeness implies that the image of $\operatorname{Pic}\left(S_{\overline{\mathbf{F}_{p}}}\right)$ in étale cohomology is the $\mathbf{Z}$-line spanned by $O(1)$. Therefore, every 
curve in $S_{\overline{\mathbf{F}_{p}}}$ has degree a multiple of 4 . So the 3 -fold $X_{\mathbf{F}_{p}}=S_{\mathbf{F}_{p}} \times \mathbf{P}^{1}$ with the very ample line bundle $L=\pi_{1}^{*} O(1) \otimes \pi_{2}^{*} O(4)$ has the property that every curve $C$ on $X_{\overline{\mathbf{F}_{p}}}$ has $L \cdot C \equiv 0(\bmod 4)$. We have arranged that the 3 -fold $X_{\mathbf{F}_{p}} \subset \mathbf{P}^{N}$ has a projection to $\mathbf{P}^{4}$, defined over $\mathbf{F}_{p}$, which is a morphism that is (set-theoretically) 2-to-1 at most in dimension two, 3-to-1 at most in dimension one, and 4-to-1 or worse at most in dimension zero. Therefore, the image of this projection is a singular hypersurface $Y$ of degree 48 in $\mathbf{P}^{4}$ over $\mathbf{F}_{p}$ such that every curve in $Y_{\overline{\mathbf{F}} p}$ has even degree.

It follows that any smooth hypersurface of degree 48 in $\mathbf{P}^{4}$ over $\overline{\mathbf{Q}}$ that specializes to $Y$ over $\overline{\mathbf{F}_{p}}$ has every curve of even degree. Since $Y$ is defined over $\mathbf{F}_{p}$, this applies to a Zariski-dense set of smooth hypersurfaces over $\mathbf{Q}$.

\section{Abelian 3-folds}

As discussed in Section 2, Griffiths and Harris conjectured that every curve in a very general complex 3 -fold of degree $d \geq 6$ in $\mathbf{P}^{4}$ has degree a multiple of $d$. The best result towards Griffiths and Harris's conjecture for hypersurfaces of high degree is due to Debarre et al. [7]. Namely, for every odd $k$ at least 9, if $X$ is a very general complex hypersurface of degree $6 k$, then every curve on $X$ has degree divisible by $k$. We produce hypersurfaces over the rational numbers with similar properties.

THEOREM 6.1. For any integer $k$ prime to 6 and at least 38, there is a smooth hypersurface $X$ of degree $6 k$ in $\mathbf{P}^{4}$ over $\mathbf{Q}$ such that every curve in $X_{\overline{\mathbf{Q}}}$ has degree divisible by $k$. The set of such hypersurfaces over $\mathbf{Q}$ is Zariski dense in the space of all hypersurfaces of degree $6 k$.

Debarre, Hulek, and Spandaw's method combines Kollár's lemma with the fact that a very general $(1,1, k)$-polarized complex abelian 3-fold has Picard number 1 . In order to apply Lemma 4.1, we would like to find a $(1,1, k)$-polarized abelian 3 -fold over $\overline{\mathbf{F}_{p}}$ with Picard number 1 , but in fact every abelian variety of dimension $g$ over $\overline{\mathbf{F}_{p}}$ has Picard number at least $g$, as a consequence of Tate's theorem that every abelian variety over $\overline{\mathbf{F}_{p}}$ is of complex multiplication type [29]. We can get around the problem at the cost of the slightly weakened statement in Theorem 6.1.

Proof. We use a special case of Kollár's refinement of Angehrn and Siu's effective basepoint-freeness theorem. Let $X$ be a smooth complex projective 3 -fold with an ample line bundle $L$. Suppose that $L \cdot C \geq 38$ for every curve $C$ on $X, L^{2} \cdot S \geq 76$ for every surface $S$ on $X$, and $L^{3} \geq 218$. Then the line bundle $K_{X} \otimes L$ is basepoint free, and the associated morphism $X \rightarrow \mathbf{P}^{N}$ is injective [18, Theorem 
5.9]. The results available today would require stronger assumptions in order to make the derivative of this morphism injective, but we will not need that.

By definition, an ample line bundle $L$ on an abelian 3 -fold $X$ gives a $(1,1, k)$-polarization if the associated homomorphism $X \rightarrow \widehat{X}=\operatorname{Pic}^{0}(X), x \mapsto$ $t_{x}^{*} L \otimes L^{-1}$, has kernel isomorphic to $(\mathbf{Z} / k)^{2}[3$, Section 3.1]. Such a line bundle has $L^{3}=6 k$. It follows that, for all $k \geq 38$, if $(X, L)$ is a $(1,1, k)$-polarized complex abelian 3 -fold such that every curve $C$ on $X$ has $L \cdot C \equiv 0(\bmod k)$ and every surface $S$ on $X$ has $L^{2} \cdot S \equiv 0(\bmod 2 k)$, then $L$ is basepoint free and the associated morphism $X \rightarrow \mathbf{P}^{N}$ is injective. A very general $(1,1, k)$-polarized abelian 3 -fold satisfies these and even stronger properties; for example, $L \cdot C \equiv 0(\bmod 3 k)$ for every curve $C$ on $X$ [16]. For our application to abelian 3 -folds over finite fields, however, we have to consider a special class of abelian 3-folds.

Let $E_{1}, E_{2}, E_{3}$ be elliptic curves, and let $\epsilon_{j}$ be a point of order $k$ on $E_{j}$ for $j=1,2,3$. Let $X=\left(E_{1} \times E_{2} \times E_{3}\right) /(\mathbf{Z} / k)^{2}$ be the quotient by the subgroup generated by $\epsilon_{j}-\epsilon_{k}$ for $1 \leq i<j \leq 3$. By Debarre, Hulek, and Spandaw, there is an ample line bundle $L$ of type $(1,1, k)$ on $X$ whose pullback to $E_{1} \times E_{2} \times E_{3}$ is $\pi_{1}^{*} O(k[0]) \otimes \pi_{2}^{*} O(k[0]) \otimes \pi_{3}^{*} O(k[0])[7$, proof of Proposition 2].

For very general complex elliptic curves $E_{i}, A:=E_{1} \times E_{2} \times E_{3}$ has Picard number 3, with Néron-Severi group generated by the divisors $0 \times E_{2} \times E_{3}$, $E_{1} \times 0 \times E_{3}$, and $E_{1} \times E_{2} \times 0$. It follows that the subgroup of Hodge classes in $H_{2}\left(E_{1} \times E_{2} \times E_{3}, \mathbf{Z}\right)$ also has rank three, spanned by the curves $E_{1} \times 0 \times 0$, $0 \times E_{2} \times 0$, and $0 \times 0 \times E_{3}$. It also follows that $X$ has Picard number 3 , and we can compute the group $N^{1}(X) \cong \mathbf{Z}^{3}$ of Hodge classes in $H^{2}(X, \mathbf{Z})$. For a suitable basis $x_{1}, y_{1}, x_{2}, y_{2}, x_{3}, y_{3}$ for $H^{1}(A, \mathbf{Z})$ coming from the product decomposition $A=E_{1} \times E_{2} \times E_{3}$, the isogeny $\pi: A \rightarrow X$ makes $\pi^{*}: H^{1}(X, \mathbf{Z}) \rightarrow H^{1}(A, \mathbf{Z})$ injective with image spanned over $\mathbf{Z}$ by $x_{1}, k y_{1}, x_{2}, k y_{2}, x_{3}, y_{1}+y_{2}+y_{3}$. Since $H^{j}(A, \mathbf{Z})=\Lambda^{j} H^{1}(A, \mathbf{Z})$ and likewise for $X$, we can compute $H^{j}(X, \mathbf{Z})$ as a subgroup of $H^{j}(A, \mathbf{Z})$ for each $j$. In particular, the Néron-Severi group $N^{1}(A)$ is spanned by $x_{1} \wedge y_{1}, x_{2} \wedge y_{2}, x_{3} \wedge y_{3}$, and we read off that $N^{1}(X)$ is spanned by $k\left(x_{1} \wedge y_{1}\right), k\left(x_{2} \wedge y_{2}\right), k\left(x_{3} \wedge y_{3}\right)$. In particular, $L \in N^{1}(X)$ pulls back to $k\left(x_{1} \wedge y_{1}\right)+k\left(x_{2} \wedge y_{2}\right)+k\left(x_{3} \wedge y_{3}\right)$.

The pushforward map $\pi_{*}: H_{2}(A, \mathbf{Z}) \rightarrow H_{2}(X, \mathbf{Z})$ is dual to $\pi^{*}: H^{2}(X, \mathbf{Z}) \rightarrow$ $H^{2}(A, \mathbf{Z})$, and so it can also be computed explicitly. In particular, we find that $\pi_{*}$ gives an isomorphism from the Hodge classes in $H_{2}(A, \mathbf{Z})$ to the Hodge classes in $H_{2}(X, \mathbf{Z})$. It follows that every curve $C$ on $X$ has $L \cdot C \equiv 0(\bmod k)$. Likewise, using that

$$
L^{2}=2 k^{2}\left(x_{1} \wedge y_{1} \wedge x_{2} \wedge y_{2}+x_{1} \wedge y_{1} \wedge x_{3} \wedge y_{3}+x_{2} \wedge y_{2} \wedge x_{3} \wedge y_{3}\right),
$$


together with the calculation that $N^{1}(X)=\mathbf{Z}\left\{k\left(x_{1} \wedge y_{1}\right), k\left(x_{2} \wedge y_{2}\right), k\left(x_{3} \wedge y_{3}\right)\right\}$ and the fact that $A \rightarrow X$ has degree $k^{2}$, we have $L^{2} \cdot S \equiv 0(\bmod 2 k)$ for every surface $S$ on $X$.

By the basepoint-freeness results mentioned earlier, these congruences imply that, for $k \geq 38$ and very general complex elliptic curves $E_{1}, E_{2}, E_{3}$, the $(1,1, k)$-polarized abelian 3-fold $(X, L)$ with $X=\left(E_{1} \times E_{2} \times E_{3}\right) /(\mathbf{Z} / k)^{2}$ has $L$ basepoint free, and the resulting morphism to projective space is injective. The conclusion is a Zariski open condition, and so the same conclusion holds for general (rather than very general) complex elliptic curves $E_{1}, E_{2}, E_{3}$.

Let $Y_{1}(k)$ be the moduli scheme over $\mathbf{Z}[1 / k]$ of elliptic curves with a point of order $k$, and let $E \rightarrow Y_{1}(k)$ be the corresponding universal family [15, Corollary 2.7.3, Theorem 3.7.1, Corollary 4.7.1]. Let $B$ be the fiber product $Y_{1}(k)^{3}$ over $\mathbf{Z}$. Then we have a family $A \rightarrow B$ of abelian 3-folds with fibers of the form $E_{1} \times E_{2} \times E_{3}$. Using the given points of order $k$ on $E_{1}, E_{2}, E_{3}$, we also have a family $X \rightarrow B$ of abelian 3-folds with $(1,1, k)$-polarization $L$, where the fibers are of the form $\left(E_{1} \times E_{2} \times E_{3}\right) /(\mathbf{Z} / k)^{2}$.

Let $X_{\mathbf{Q}(B)}$ be the generic fiber of $X \rightarrow B$, which is an abelian 3-fold over the function field $\mathbf{Q}(B)$. By our work over $\mathbf{C}$, for $k \geq 38, L$ is basepoint free on $X_{\mathbf{Q}(B)}$, of degree $L^{3}=6 k$, and the associated morphism $X_{\mathbf{Q}(B)} \rightarrow \mathbf{P}^{N}$ is injective. By Lemma 4.3, applying a general projection to $\mathbf{P}^{4}$ gives a morphism $X_{\mathbf{Q}(B)} \rightarrow \mathbf{P}^{4}$ that is (set-theoretically over $\overline{\mathbf{Q}(B)}$ ) 2-to-1 at most in dimension two, 3-to-1 at most in dimension one, and 4-to-1 or worse at most in dimension zero. We can view this morphism as a rational map $X \rightarrow \mathbf{P}_{B}^{4}$ over $B$. After replacing $B$ by some nonempty open subset, we have a morphism $X \rightarrow \mathbf{P}_{B}^{4}$ over $B$ which is 2-to-1 at most in dimension two, and so on, over every point of $B$.

Let $l$ be a prime number. The arithmetic fundamental group $\pi_{1} B$ (with a base point in $B(\overline{\mathbf{Q}}))$ acts on $H^{1}\left(A_{\overline{\mathbf{Q}}}, \mathbf{Z}_{l}\right) \cong \mathbf{Z}_{l}^{6}$. Here, $H^{1}\left(A_{\overline{\mathbf{Q}}}, \mathbf{Q}_{l}\right)=H^{1}\left(E_{1} \times E_{2} \times\right.$ $\left.E_{3}, \mathbf{Q}_{l}\right)=V_{1} \oplus V_{2} \oplus V_{3}$, where $V_{i}$ is the standard representation of the $i$ th copy of $G L\left(2, \mathbf{Q}_{l}\right)$. As a representation of $\pi_{1} B$, all three representations $V_{i}$ have the same determinant $\mathbf{Q}_{l}(-1)$, by Poincaré duality. Because the geometric fundamental group $\pi_{1} B_{\overline{\mathbf{Q}}}$ is Zariski dense in $\operatorname{SL}\left(2, \mathbf{Q}_{l}\right)^{3}$, the arithmetic fundamental group $\pi_{1} B$ is Zariski dense in the algebraic group $G=G_{m} \cdot \operatorname{SL}(2)^{3}$ over $\mathbf{Q}_{l}$. Next, consider the representation

$$
\begin{aligned}
H^{2}\left(A_{\overline{\mathbf{Q}}}, \mathbf{Q}_{l}(1)\right) & \cong\left(\Lambda^{2} H^{1}\left(A_{\overline{\mathbf{Q}}}, \mathbf{Q}_{l}\right)\right)(1) \\
& \cong\left(\mathbf{Q}_{l}\right)^{3} \oplus\left(V_{1} \otimes V_{2}\right)(1) \oplus\left(V_{1} \otimes V_{3}\right)(1) \oplus\left(V_{2} \otimes V_{3}\right)(1) .
\end{aligned}
$$

Most elements of $G\left(\mathbf{Q}_{l}\right)$ have no eigenvalues which are roots of unity on $\left(V_{i} \otimes V_{j}\right)(1)$, for $i \neq j$ in $\{1,2,3\}$. Using the Serre-Chebotarev equidistribution theorem as in the proof of Lemma 5.1, it follows that there is a closed point $x$ of $B$ with residue field of prime order such that the corresponding abelian 3-fold 
$A_{\mathbf{F}_{p}}=E_{1} \times E_{2} \times E_{3}$ has geometric Picard number 3 . Thus there are elliptic curves $E_{1}, E_{2}, E_{3}$ over $\mathbf{F}_{p}$ such that the abelian 3 -fold $X=\left(E_{1} \times E_{2} \times E_{3}\right) /(\mathbf{Z} / k)^{2}$ has Picard number 3 over $\overline{\mathbf{F}_{p}}$, while (by our shrinking of the base space $B$ ) a sublinear system of the $(1,1, k)$-polarization $|L|$ gives a morphism $X \rightarrow \mathbf{P}^{4}$ over $\mathbf{F}_{p}$ which is (set-theoretically over $\overline{\mathbf{F}_{p}}$ ) 2-to-1 at most in dimension two, 3-to-1 at most in dimension one, and 4-to-1 or worse at most in dimension zero.

We have $L^{3}=6 k$. By the description of the pullback map $H^{*}\left(X, \mathbf{Z}_{l}\right) \rightarrow$ $H^{*}\left(A, \mathbf{Z}_{l}\right)$ given earlier in the case of complex abelian 3-folds, which we apply for the prime factors $l$ of $k$, it follows from $\mathrm{X}$ having geometric Picard number 3 that every curve $C$ on $X_{\overline{\mathbf{F}_{p}}}$ has $L \cdot C \equiv 0(\bmod k)$. Let $Y$ be the singular hypersurface of degree $6 k$ in $\mathbf{P}^{4}$ over $\mathbf{F}_{p}$ which is the image of $X$. Then every curve $C$ in $Y_{\overline{\mathbf{F}_{p}}}$ satisfies $6 \operatorname{deg}(C) \equiv 0(\bmod k)$. Therefore, every smooth hypersurface $X$ of degree $6 k$ in $\mathbf{P}^{4}$ over $\overline{\mathbf{Q}}$ that specializes to $Y_{\overline{\mathbf{F}_{p}}}$ has the property that every curve $C$ in $X$ satisfies $6 \operatorname{deg}(C) \equiv 0(\bmod k)$. Since $Y$ is defined over $\mathbf{F}_{p}$, this applies to a Zariski-dense set of hypersurfaces of degree $6 k$ over $\mathbf{Q}$. In the special case where $k$ is prime to 6 , the statement that $6 \operatorname{deg}(C) \equiv 0(\bmod k)$ implies that $\operatorname{deg}(C) \equiv 0(\bmod k)$.

\section{Acknowledgements}

I thank the excellent referees for several corrections and references. The article processing charge was paid by NSF grant DMS-1303105.

\section{References}

[1] M. Artin, 'Supersingular K3 surfaces', Ann. Sci. Éc. Norm. Supér. 7 (1974), 543-567.

[2] M. Atiyah and F. Hirzebruch, 'Analytic cycles on complex manifolds', Topology 1 (1962), $25-45$.

[3] C. Birkenhake and H. Lange, Complex Abelian Varieties (Springer, 2004).

[4] F. Charles, The Tate conjecture for K3 surfaces over finite fields. http://arxiv.org/abs/1206. 4002.

[5] J.-L. Colliot-Thélène and T. Szamuely, 'Autour de la conjecture de Tate à coefficients $\mathbf{Z}_{l}$ pour les variétés sur les corps finis', in The Geometry of Algebraic Cycles (AMS/Clay Institute Proceedings, 2010), 83-98.

[6] J.-L. Colliot-Thélène and C. Voisin, 'Cohomologie non ramifiée et conjecture de Hodge entière', Duke Math. J. 161 (2012), 735-801.

[7] O. Debarre, K. Hulek and J. Spandaw, 'Very ample linear systems on abelian varieties', Math. Ann. 300 (1994), 181-202.

[8] P. Deligne, 'La conjecture de Weil. I', Publ. Math. Inst. Hautes Études Sci. 43 (1974), 273-307. 
[9] P. Deligne, 'La conjecture de Weil. II', Publ. Math. Inst. Hautes Études Sci. 52 (1980), 137-252.

[10] A. J. de Jong and N. Katz, 'Monodromy and the Tate conjecture: Picard numbers and Mordell-Weil ranks in families', Israel J. Math. 120 (2000), 47-79.

[11] W. Fulton, Intersection Theory (Springer, 1984).

[12] C. Grabowski, On the integral Hodge conjecture for 3-folds. PhD Thesis, Duke University (2004).

[13] P. Griffiths and J. Harris, Principles of Algebraic Geometry (Wiley, 1978).

[14] P. Griffiths and J. Harris, 'On the Noether-Lefschetz theorem and some remarks on codimension two cycles', Math. Ann. 271 (1985), 31-51.

[15] N. Katz and B. Mazur, Arithmetic Moduli of Elliptic Curves (Princeton, 1985).

[16] J. Kollár, 'Trento examples', in Classification of Irregular Varieties (Trento, 1990), Lecture Notes in Mathematics, vol. 1515 (Springer, 1992), 134-135.

[17] J. Kollár, Rational Curves on Algebraic Varieties (Springer, 1996).

[18] J. Kollár, 'Singularities of pairs', Proc. Symp. Pure Math. 62, Part 1 (Amer. Math. Soc., 1997), 221-287.

[19] D. Maulik, Supersingular K3 surfaces for large primes. http://arxiv.org/abs/1203.2889.

[20] K. Madapusi Pera, The Tate conjecture for K3 surfaces in odd characteristic. http://arxiv. org/abs/1301.6326.

[21] J. Milne, Étale Cohomology (Princeton, 1980).

[22] M. Nori, 'Algebraic cycles and Hodge-theoretic connectivity', Invent. Math. 111 (1993), 349-373.

[23] N. Nygaard and A. Ogus, 'Tate's conjecture for K3 surfaces of finite height', Ann. of Math. (2) 122 (1985), 461-507.

[24] J. Rathmann, 'The uniform position principle for curves in characteristic p', Math. Ann. 276 (1987), 565-579.

[25] C. Schoen, 'An integral analog of the Tate conjecture for one-dimensional cycles on varieties over finite fields', Math. Ann. 311 (1998), 493-500.

[26] J.-P. Serre, 'Zeta and L functions', in Arithmetical Algebraic Geometry (Purdue, 1963) (Harper \& Row, 1965), 82-92.

[27] T. Shioda, 'Algebraic cycles on a certain hypersurface', in Algebraic Geometry (Tokyo/Kyoto, 1982), Lecture Notes in Mathematics, 1016 (Springer, 1983), 271-294.

[28] C. Soulé and C. Voisin, 'Torsion cohomology classes and algebraic cycles on complex projective manifolds', Adv. Math. 198 (2005), 107-127.

[29] J. Tate, 'Endomorphisms of abelian varieties over finite fields', Invent. Math. 2 (1966), 134-144.

[30] B. Totaro, 'Torsion algebraic cycles and complex cobordism', J. Amer. Math. Soc. 10 (1997), 467-493.

[31] B. Totaro, 'Non-injectivity of the map from the Witt group of a variety to the Witt group of its function field', J. Inst. Math. Jussieu 2 (2003), 483-493.

[32] C. Voisin, 'On integral Hodge classes on uniruled and Calabi-Yau threefolds', in Moduli Spaces and Arithmetic Geometry, Advanced Studies in Pure Mathematics, 45 (2006), 43-73. 